\title{
Prevalencia de las deficiencias de zinc y cobre en adultos mayores de la Región Metropolitana de Santiago
}

\author{
MANUEL OLIVARES, LYDIA LERA ${ }^{\text {a }}$ CECILIA ALBALA, \\ FERNANDO PIZARRO ${ }^{\mathrm{b}}$, MAGDALENA ARAYA
}

\section{Prevalence of zinc and copper deficiencies in older subjects living in Metropolitan Santiago}

Background: Older subjects have a high risk of developing zinc and copper deficiencies. Aim: To determine the prevalence of copper and zinc deficiencies in adults aged over 60 years, living in Metropolitan Santiago. Subjects and Methods: Analyses were performed using anonymous serum samples from older subjects living in Santiago, Chile, who participated in the multicenter project SABE. Of the studied subjects, $49.3 \%$ had enough left-over serum to measure serum zinc and copper concentrations by atomic absorption spectrometry. Results: A total of 444 men and 198 women were studied. A positive correlation between serum zinc and copper was found in both women $(r=0.44, p<0.001)$ and men $(r=0.48, p<0.001)$. There were no differences in serum zinc concentration by sex and age. The prevalence of subnormal serum zinc values was $66.9 \%$ in women and $66.7 \%$ in men. Women had a significantly higher serum copper values than men $(p<0.02)$ and there were no differences by age. Prevalence of subnormal serum copper values was higher in women than in men (32.9 and $23.7 \%$ respectively, $p<0.05$ ). Women had significantly higher copper/zinc serum ratio than men $(p<0.001)$. Conclusions: Older people living in Metropolitan Santiago have a high prevalence of copper and zinc deficiencies.

(Rev Med Chile 2011; 139: 283-289).

Key words: Aged; Copper; Chile; Nutritional status; Zinc.

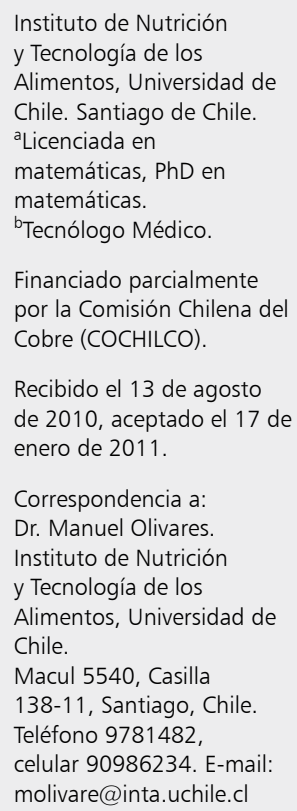

E 1 zinc $(\mathrm{Zn})$ y el cobre $(\mathrm{Cu})$ poseen importantes funciones en el organismo ya que son necesarios para la función de numerosas enzimas. El primero es importante para la integridad estructural de proteínas y membranas, participa en la unión de hormonas a sus receptores y en la regulación de la expresión génica ${ }^{1}$. El segundo es necesario para procesos tales como el transporte de electrones, la dismutación de superóxidos, la síntesis de melanina, el entrecruzamiento del colágeno y elastina, la síntesis de neurotransmisores y la oxidación del hierro ${ }^{2}$. Estas funciones hacen que ambos sean nutrientes fundamentales para el adecuado funcionamiento del sistema inmune.

La prevalencia de la deficiencia de Zn no se conoce con exactitud ya que no se cuenta con indicadores de laboratorio de alta sensibilidad y confiabilidad $^{3}$. Esto ha dificultado la estimación de la real magnitud de esta deficiencia. Se estima que es un problema prevalente en los países en desarrollo y que su magnitud no sería muy distinta de la deficiencia de hierro ${ }^{3}$. La prevalencia de la deficiencia de $\mathrm{Cu}$ no se conoce bien, es menos común que la carencia de Zn, sin embargo, sería más frecuente de lo estimado previamente ${ }^{4}$.

El aumento de las expectativas de vida es un fenómeno creciente a nivel mundial que ha llevado a un aumento progresivo del número de adultos mayores. Este grupo etario es más vulnerable a experimentar deficiencias de micronutrientes 
debido a un menor consumo de alimentos por limitaciones socioeconómicas, aislamiento social, menor actividad física o por una anorexia originada por alteraciones sensoriales, consumo de algunos medicamentos o a factores psicológicos ${ }^{5,6}$. A esto se agregan dificultades en la digestión por problemas en la masticación por la pérdida de piezas dentales y en la absorción de algunos nutrientes a consecuencias de posibles alteraciones en la función gastrointestinal ${ }^{7,8}$.

Existe escasa información nacional y regional sobre la prevalencia de deficiencias de zinc y cobre en adultos mayores, especialmente de este último mineral.

El propósito del estudio es determinar la prevalencia de las deficiencias de zinc y cobre en adultos de 60 años y más de la Región Metropolitana de Santiago de Chile.

\section{Sujetos y Métodos}

El estudio se efectuó en muestras de suero que se anonimizaron y que pertenecían a sujetos de 60 años y más, de la ciudad de Santiago de Chile, que habían participado en el proyecto multicéntrico SABE (Encuesta Salud, Bienestar y Envejecimiento) realizado en 7 ciudades de América Latina y el Caribe en el año $1999^{\circ}$. Para la muestra de Santiago de Chile, se seleccionaron 1.301 sujetos viviendo en la comunidad, mediante un muestreo probabilístico por conglomerados, realizado en tres etapas, en las que se seleccionaron sucesivamente los distritos, manzanas y hogares . $^{9}$

Del total de sujetos estudiados en el proyecto SABE, a 930 se les había tomado una muestra de sangre en ayunas para la realización de exámenes rutinarios y habían dado su consentimiento para guardar una muestra congelada a $-20^{\circ} \mathrm{C}$ en criotubos para futuros estudios de nutrición. De ellos, 642 (49,3\%) tenían suficiente muestra de suero para realizar mediciones de las concentraciones de zinc y cobre séricos. El tubo con suero en el que se determinó la concentración sérica de minerales nunca antes había sido descongelado. Estos minerales se midieron por espectrometría de absorción atómica (modelo 2280, The PerkinElmer Corporation, Norwalk,CT, USA).

La muestra quedó constituida por 444 mujeres y 198 hombres de 60 y más años (Tabla 1).

Se definieron como niveles subnormales de zinc valores séricos $<70 \mu \mathrm{g} / \mathrm{dl}$ en mujeres $\mathrm{y}<74$ $\mu \mathrm{g} / \mathrm{dl}$ en hombres ${ }^{3}$ y niveles subnormales de cobre a valores séricos $<80 \mu \mathrm{g} / \mathrm{dl}$ en mujeres $\mathrm{y}<70 \mu \mathrm{g} /$ $\mathrm{dl}$ en hombres ${ }^{10}$.

La utilización de las muestras de suero almacenadas de los participantes en el proyecto SABE fue autorizada por el Comité de Ética del Instituto de Nutrición y Tecnología de los Alimentos, Universidad de Chile.

\section{Estadística}

Los datos se analizaron en base al sexo y las categorías de edad 60 a 64, 65 a 74 y $\geq 74$ años. Los resultados se expresan como promedios y desviaciones estándar. Se estudió la correlación entre niveles plasmáticos de $\mathrm{Cu}$ y $\mathrm{Zn}$ por medio de correlación simple de Pearson. Se utilizó el modelo lineal general (MLG) para evaluar las diferencias entre los grupos de las concentraciones séricas de zinc y cobre, mientras la prueba de chi-cuadrado se empleó para evaluar las diferencias en la frecuencia de valores subnormales de estos minerales. Los análisis se realizaron con el programa Stata 10.1 (StataCorp, College Station, TX, USA).

Tabla 1. Distribución porcentual de los adultos mayores por sexo y grupos de edad

\begin{tabular}{|lcccccc|}
\hline Edad (años) & \multicolumn{2}{c}{ Mujeres } & \multicolumn{2}{c}{ Hombres } & \multicolumn{2}{c|}{ Total } \\
& $\mathbf{n}$ & $\mathbf{\%}$ & $\mathbf{n}$ & $\mathbf{\%}$ & $\mathbf{n}$ & $\%$ \\
$60-64$ & 74 & 11,5 & 39 & 6,1 & 113 & 17,6 \\
$65-74$ & 187 & 29,1 & 99 & 15,4 & 286 & 44,5 \\
$\geq 75$ & 183 & 28,5 & 60 & 9,3 & 243 & 37,9 \\
Total & 444 & 69,2 & 198 & 30,8 & 642 & 100,0 \\
\hline
\end{tabular}


Tabla 2. Concentración de zinc y cobre séricos por sexo y grupos de edad

\begin{tabular}{|c|c|c|c|c|}
\hline \multirow[t]{2}{*}{ Edad (años) } & \multicolumn{2}{|c|}{ Zinc sérico $(\mu \mathrm{g} / \mathrm{dl})^{*}$} & \multicolumn{2}{|c|}{ Cobre sérico $(\mu \mathrm{g} / \mathrm{dl})^{* *}$} \\
\hline & Mujeres & Hombres & Mujeres & Hombres \\
\hline $60-64$ & $65,7 \pm 18,0$ & $68,1 \pm 15,1$ & $94,9 \pm 27,4$ & $87,2 \pm 24,7$ \\
\hline $65-74$ & $65,2 \pm 19,2$ & $70,8 \pm 19,2$ & $99,6 \pm 28,6$ & $93,9 \pm 27,4$ \\
\hline$\geq 75$ & $65,4 \pm 18,7$ & $63,0 \pm 15,6$ & $98,1 \pm 26,3$ & $92,2 \pm 27,0$ \\
\hline Total & $65,4 \pm 18,8$ & $67,9 \pm 17,6$ & $98,2 \pm 27,4$ & $92,1 \pm 26,8$ \\
\hline
\end{tabular}

Promedio y desviación estándar. $M L G=$ *Zinc sérico: sexo $F=1,15, p=0,2829$; edad $F=2,29, p=0,1017$; interacción $F=$ $2,53, p=0,0807 .{ }^{* *}$ Cobre sérico: sexo $F=6,53, p=0,0108$; edad $F=1,62, p=0,1985$; interacción $F=0,05, p=0,9483$.

Tabla 3. Porcentaje de sujetos con valores subnormales de zinc y cobre séricos por sexo y grupos de edad

\begin{tabular}{|lcccc|}
\hline Edad (años) & Zinc (\%) & \multicolumn{2}{c|}{ Cobre (\%) } \\
& Mujeres & Hombres & Mujeres & Hombres \\
$60-64$ & 66,2 & 74,4 & 44,6 & $25,6^{*}$ \\
$65-74$ & 69,0 & 59,6 & 30,0 & 22,2 \\
$\geq 74$ & 65,0 & 73,3 & 31,2 & 23,3 \\
\hline Total & 66,9 & 66,7 & 32,9 & $23,7^{* *}$ \\
\hline
\end{tabular}

${ }^{*}$ Chi-cuadrado: ${ }^{*} p<0,03 y{ }^{* *} p<0,05$ respecto a las mujeres.

\section{Resultados}

$82,4 \%$ de los 642 sujetos analizados era mayor de 64 años. Las mujeres se encontraban en una proporción de 2,2 a 1 respecto a los hombres (Tabla 1).

No se apreciaron diferencias por sexo ni edad en la concentración de zinc sérico (MLG: sexo $\mathrm{F}=1,15, \mathrm{p}=0,283$; edad $\mathrm{F}=2,29, \mathrm{p}=0,102$; interacción $\mathrm{F}=2,53, \mathrm{p}=0,081)$. El promedio $\mathrm{y}$ desviación estándar de zinc sérico en las mujeres fue $65,4 \pm 18,8 \mu \mathrm{g} / \mathrm{dl}$ y en los hombres $67,9 \pm 17,6$ $\mu \mathrm{g} / \mathrm{dl}$ (Tabla 2).

La prevalencia de valores subnormales de zinc sérico fue muy elevada en mujeres $(66,9 \%)$ y hombres $(66,7 \%)$, no existiendo diferencias significativas de acuerdo al género o edad (Tabla 3).

Las mujeres presentaron valores séricos de cobre significativamente más elevados que los hombres $(98,2 \pm 27,4 \mu \mathrm{g} / \mathrm{dl}$ vs $92,1 \pm 26,8 \mu \mathrm{g} / \mathrm{dl})$ (Tabla 2), no existiendo diferencias por tramo de edad (MLG: sexo $\mathrm{F}=6,53, \mathrm{p}=0,011$; edad $\mathrm{F}=$ $1,62, \mathrm{p}=0,199$; interacción $\mathrm{F}=0,05, \mathrm{p}=0,948$ ).
La prevalencia de valores subnormales de cobre sérico fue más alta en mujeres que en hombres $(32,9$ vs $23,7 \%$, Chi-cuadrado $\mathrm{p}<0,05)$ (Tabla $3)$. No se observaron diferencias en la prevalencia de deficiencia de cobre de acuerdo a la edad en hombres ni en mujeres.

Se encontró una correlación positiva entre los valores de zinc y cobre séricos en mujeres $(\mathrm{r}=$ $0,44, p<0,001)$ y hombres $(r=0,48, p<0,001)$.

Las mujeres tuvieron una proporción cobre/ zinc en suero de $1,57 \pm 0,50$ y los hombres 1,40 $\pm 0,40$ (MLG: sexo $\mathrm{F}=15,08, \mathrm{p}=0,001$; edad $\mathrm{F}=$ $2,89, \mathrm{p}=0,057$; interacción $\mathrm{F}=1,77, \mathrm{p}=0,172)$.

\section{Discusión}

Este es el primer estudio que determina la prevalencia de las deficiencias de zinc y cobre en una muestra representativa de adultos mayores de la Región Metropolitana de Santiago, Chile. En nuestro estudio encontramos una elevada prevalencia de sujetos deficientes en zinc $(66,8 \%)$ 
y cobre $(30,1 \%)$. Cabe señalar que las muestras sanguíneas se tomaron una década atrás, sin bien las mediciones de zinc y cobre se realizaron recientemente en las muestras de suero almacenadas, las que se mantuvieron selladas y refrigeradas a $-20^{\circ} \mathrm{C}$, por lo que desconocemos la prevalencia actual de deficiencias de estos microminerales, información que sería de alto interés contar con ella.

$\mathrm{Al}$ analizar la prevalencia de estas deficiencias según género no hubo diferencias en la carencia de zinc, sin embargo, la carencia de cobre fue más elevada en mujeres $(32,9 \%)$ que en hombres $(23,7 \%)$. No se apreció una variación de la prevalencia de la deficiencia de zinc o de cobre relacionada con la edad, contrariamente a lo observado en otros estudios que han descrito un aumento de la prevalencia de zinc en ancianos a medida que se incrementa la edad $^{11} \mathrm{o}$ están hospitalizados ${ }^{12}$, lo que probablemente se explicaría porque que nuestros adultos mayores residían en sus hogares, eran autovalentes y no tenían restricciones relacionadas con la edad en el acceso a alimentos fuentes de zinc. Otros estudios realizados en adultos mayores de nuestra región han mostrado también altas prevalencias de deficiencia de zinc ${ }^{13,14}$ o zinc y cobre ${ }^{15}$.

Al igual que la mayoría de los estudios, nosotros definimos como deficientes a aquellos sujetos que tenían valores séricos subnormales de los nutrientes estudiados. Cabe señalar que las concentraciones séricas de zinc y de cobre están fuertemente reguladas y sólo se alteran en deficiencias moderadas a severas de estos oligoelementos y por tanto no son útiles para detectar una deficiencia leve de estos micronutrientes ${ }^{3,16}$. Teniendo en cuenta este hecho, la prevalencia de las deficiencias de zinc y de cobre serían aun más prevalentes en el grupo estudiado. Por otra parte, los procesos inflamatorios, condición que es más frecuente en adultos mayores, alteran el metabolismo de ambos nutrientes, produciendo un descenso de los valores séricos de zinc y un aumento de los niveles séricos de cobre ${ }^{17,18}$, de modo que pudiera estar sobrestimada la prevalencia de deficiencia de zinc y subestimada la prevalencia de la deficiencia de cobre. En un estudio previo, encontramos que alrededor de $11 \%$ de ancianos que vivían en sus hogares presentaban evidencias de laboratorio de inflamación ${ }^{19}$. Por otra parte, cabe preguntarse si es válido aplicar en adultos mayores los mismos puntos de corte utilizados en adultos jóvenes para definir valores subnormales de zinc y cobre séricos. Si bien no hay consenso, existen estudios que encuentran que los adultos mayores tienen valores de zinc sérico más bajos y de cobre sérico más elevados que los encontrados en adultos jóvenes ${ }^{3,20-23}$. Se ha descrito también un aumento de la proporción $\mathrm{Cu} / \mathrm{Zn}$ séricos en adultos mayores a medida que progresa la edad ${ }^{24}$. La pregunta aún sin resolver es en qué medida esta reducción de los valores séricos de zinc sería consecuencia de los cambios fisiológicos que ocurren durante el envejecimiento, como por ejemplo, la reducción de las masas muscular y ósea, que contienen parte importante del zinc tisular y cuánto se debería a que este grupo etario presenta una mayor frecuencia de procesos inflamatorios y una mayor prevalencia de deficiencia de zinc. Si en los adultos mayores fuera necesario utilizar puntos de corte más bajos de zinc sérico, la prevalencia de la deficiencia de zinc estaría sobrestimada en nuestro estudio. Los valores séricos de cobre más elevados que se encuentran en adultos mayores parecen más deberse al efecto de los procesos inflamatorios que a cambios fisiológicos que ocurren con la edad. Cabe señalar que la proporción $\mathrm{Cu} / \mathrm{Zn}$ sérico observada en nuestros sujetos es semejante a la encontrada en sujetos afectados de patologías inflamatorias ${ }^{12,25}$, de manera que la prevalencia de la deficiencia de cobre pudiera estar subestimada en nuestra investigación.

Estudios realizados en distintos países muestran que los adultos mayores constituyen un grupo que presenta una elevada prevalencia de deficiencia de zinc, especialmente en los grupos más pobres, en aquellos que se encuentran institucionalizados u hospitalizados y en los países en vías de desarrollo ${ }^{11-15,26-28}$. Sin embargo, la información referente a la prevalencia de la deficiencia de cobre es prácticamente inexistente. En nuestro país hay una insuficiente información acerca de la prevalencia de la deficiencia de zinc y cobre en adultos mayores. A inicio de la década 1990-99, encontramos una prevalencia de deficiencia de cobre de $5,1 \%$ en mujeres y $6,5 \%$ en hombres en un estudio realizado en adultos mayores de 60 años que vivían en sus hogares en una comuna de la ciudad de Santiago de Chile ${ }^{29}$. Esta gran diferencia en la prevalencia de deficiencia de cobre respecto al actual estudio, se explicaría por el hecho que esa investigación se realizó en una muestra de conveniencia de adultos mayores que tenían una mejor adecuación de la ingesta de cobre que la 
que observamos en una muestra representativa de la ciudad de Santiago contemporánea con el actual estudio ${ }^{19}$.

La disminución de la ingesta es la principal etiología de las deficiencias de zinc y cobre en los adultos mayores. Diversos estudios nacionales $^{19,30,31}$, regionales ${ }^{13,15}$, otros países en vías de desarrollo ${ }^{27}$ e incluso de países desarrollados ${ }^{26,28,32,33}$ han mostrado una ingesta inadecuada de zinc en los adultos mayores, acentuándose la reducción de la ingesta con la edad ${ }^{5}$. Por otra parte, una ingesta insuficiente de cobre en este grupo etario ha sido demostrada en estudios nacionales ${ }^{19,29} \mathrm{y}$ algunos realizados en países desarrollados ${ }^{32,34}$. En una muestra representativa de la población de Santiago, Chile, encontramos en adultos mayores de 60 años que 86,4\% de los hombres y 57,9\% de las mujeres tenía una ingesta de zinc menor al requerimiento promedio estimado de zinc, siendo esta frecuencia para el cobre de $7,7 \%$ en hombres y $21,1 \%$ en mujeres ${ }^{19}$. Por otra parte, la densidad energética de estos nutrientes era igual a la obtenida en adultos más jóvenes, lo que evidenciaba que esta insuficiencia en el aporte de zinc y cobre se debía a una reducción en la ingesta de alimentos y no a cambios en la composición de la dieta. En adultos mayores de 60 años, 43,9\% de la ingesta de zinc y $16,8 \%$ de la ingesta de cobre provenía de carnes de animales de granja y los cereales y leguminosas aportaban 32,5\% y 42,4\% de la ingesta de zinc y cobre, respectivamente ${ }^{19}$.

Contribuiría al desarrollo de la deficiencia de zinc en ancianos la reducción de la absorción de este mineral que ocurre durante el envejecimiento. Tres estudios han demostrado una disminución de la absorción en este período de la vida ${ }^{35-37}$ y sólo uno encontró que no había cambios con la $\operatorname{edad}^{38}$. Sin embargo, cabe señalar que Turnlund y cols $^{36}$ encontraron que si bien estaba disminuida la absorción también estaban reducidas las pérdidas endógenas de este elemento, por lo que el balance de zinc era comparable al obtenido en adultos jóvenes. Por el contrario, la absorción de cobre no se afecta con la edad ${ }^{23,35,39}$.

Las consecuencias sobre la salud de la deficiencia de zinc y cobre, se producen fundamentalmente como consecuencia de la alteración del sistema inmune $e^{2,4,15,40,41}$. En el adulto mayor la situación se hace crítica considerando la disregulación del sistema inmune propia del proceso de envejecimiento $^{42}$, lo que favorecería el aumento de la morbilidad y mortalidad de este grupo poblacional ${ }^{15}$.

En conclusión, los adultos mayores chilenos estudiados tienen una elevada prevalencia de deficiencias de zinc y cobre, lo que podría incrementar la morbi mortalidad de este grupo poblacional. Considerando el papel de estos nutrientes en la función inmune, su óptimo estatus en las personas mayores debiera tener un positivo impacto en la salud pública. Ello hace necesario establecer estrategias destinadas a prevenir estas deficiencias mediante modificaciones de la dieta y suplementación o fortificación de los alimentos con estos minerales y evaluar la eficacia y efectividad de las intervenciones nutricionales presentes y futuras en este grupo poblacional.

\section{Referencias}

1. McCall KA, Huang C, Fierke CA. Function and mechanism of zinc metalloenazymes. J Nutr 2000; 130: 1437S$46 \mathrm{~S}$.

2. Uauy R, Olivares M, González M. Essentiality of copper in humans. Am J Clin Nutr 1998; 67: 952S-9S.

3. International Zinc Nutrition Consultative Group (IZINCG). Technical document \#1. Assessment of the risk of zinc deficiency in populations and options for its control. Food Nutr Bull 2004; 25: S91-204.

4. Madsen E, Gitlin JD. Copper deficiency. Curr Opin Gastroenterol 2007; 23: 187-92.

5. Wakimoto P, Block G. Dietary intake, dietary patterns, and change with age: an epidemiological perspective. J Gerontol A Biol Sci Med Sci 2001; 56: 65-80.

6. Hickson M. Malnutrition and ageing. Postgrad Med J 2006; 82: 2-8.

7. Russell RM. Changes in gastrointestinal function attributed to aging. Am J Clin Nutr 1992; 55: 1203S-7S.

8. Salles N. Basic mechanisms of the aging gastrointestinal tract. Dig Dis 2007; 25: 112-7.

9. Albala C, Lebrão ML, León Díaz EM, Ham-Chande R, Hennis AJ, Palloni A, et al. Encuesta Salud, Bienestar y Envejecimiento (SABE): metodología de la encuesta y perfil de la población estudiada. Rev Panam Salud Publica 2005; 17: 307-22.

10. Elin RJ. Reference intervals and laboratory values. En: Cecil Textbook of Medicine. $22^{\text {th }}$ ed. Goldman L, Ansiello DA, editors. Philadelphia, PA: WB Saunders; 2004. p. 2496-505.

11. Sfar S, Jawed A, Braham H, Amor S, Laporte F, Kerkeni A. Zinc, copper and antioxidant enzyme activities in 
healthy elderly Tunisian subjects. Exp Gerontol 2009; 44: 812-7.

12. Belbraouet S, Biaudet H, Tébi A, Chau N, Gray-Donald $\mathrm{K}$, Debry G. Serum zinc and copper status in hospitalized vs healthy elderly subjects. J Am Coll Nutr 2007; 26: 650-4.

13. Cruz E, Sánchez E, Paredes MC, Álvarez D, Céspedes EM. Consumo y niveles séricos de micronutrientes en adultos mayores de un consultorio médico de familia. Rev Cuba Salud Pública 2003; 29: 117-20.

14. Meertens L, Ramos N, Solano L, Llovera D, Portillo Z. Cinc sérico y subpoblaciones de linfocitos T CD4 $(+)$ y $\mathrm{CD} 8(+)$ en adultos mayores institucionalizados. Acta Cient Venez 2005; 56: 120-5.

15. Hamer DH, Sempértegui F, Estrella B, Tucker KL, Rodríguez A, Egas J, et al. Micronutrient deficiencies are associated with impaired immune response and higher burden of respiratory infections in elderly Ecuadorians. J Nutr 2009; 139: 113-9.

16. Olivares M, Méndez MA, Astudillo PA, Pizarro F. Present situation of biomarkers for copper status. Am J Clin Nutr 2008; 88: 859S-62S.

17. Oakes EJ, Lyon TD, Duncan A, Gray A, Talwar D, O'Reilly DS. Acute inflammatory response does not affect erythrocyte concentrations of copper, zinc and selenium. Clin Nutr 2008; 27: 115-20.

18. Galloway P, McMillan DC, Sattar N. Effect of the inflammatory response on trace element and vitamin status. Ann Clin Biochem 2000; 37: 289-97.

19. Olivares M, Pizarro F, De Pablo S, Araya M, Uauy R. Iron, zinc and copper: contents in common Chilean foods and daily intakes in Santiago City, Chile. Nutrition 2004; 20: 205-12.

20. Lindeman RD, Clark ML, Colmore JP. Influence of age and sex on plasma and red cell zinc concentrations. J Gerontol 1971; 26: 358-63.

21. Madari A, Ginter E, Kadrabová J. Serum copper, zinc and copper/zinc ratio in males: influence of aging. Physiol Res 1994; 43: 107-11.

22. Milne DB, Johnson PE. Assessment of copper status: effect of age and gender on reference ranges in healthy adults. Clin Chem 1993; 39: 883-7.

23. Johnson PE, Milne DB, Lykken GI. Effects of age and sex on copper absorption, biological half-life, and status in humans. Am J Clin Nutr 1992; 56: 917-25.

24. Malavolta M, Giacconi R, Piacenza F, Santarelli L, Cipriano C, Costarelli L, et al. Plasma copper/zinc ratio: an inflammatory/nutritional biomarker as predictor of all-cause mortality in elderly population. Biogerontology 2010; 11: 309-19.

25. Cheraskin E, Carpenter JH, Riordan HD. Differences in human serum copper and zinc levels in healthy and patient populations. Med Hypotheses 1986; 20: 79-85.

26. Wagner PA, Krista ML, Bailey LB, Christakis GJ, Jernigan JA, Araujo PE, et al. Zinc status of elderly black Americans from urban low-income households. Am J Clin Nutr 1980; 33: 1771-7.

27. Oldewage-Theron WH, Samuel FO, Venter CS. Zinc deficiency among the elderly attending a care centre in Sharpeville, South Africa. J Hum Nutr Diet 2008; 21: 566-74.

28. de Jong N, Gibson RS, Thomson CD, Ferguson EL, McKenzie JE, Green TJ, et al. Selenium and zinc status are suboptimal in a sample of older New Zealand women in a community-based study. J Nutr 2001; 131: 2677-84.

29. Olivares M, Hertrampf E, Capurro MT, Wegner D. Prevalence of anemia in elderly subjects living at home: role of micronutrient deficiency and inflammation. Eur J Clin Nutr 2000; 54: 834-9.

30. Urteaga C, Ramos RI, Atalah E. Validación del criterio de evaluación nutricional global del adulto mayor. Rev Med Chile 2001; 129: 871-6.

31. Atalah E, Benavides X, Avila L, Barahona S, Cárdenas R. Características alimentarias de adultos mayores de comunas pobres de la Región Metropolitana. Rev Med Chile 1998; 126: 489-96.

32. Bailey AL, Maisey S, Southon S, Wright AJ, Finglas PM, Fulcher RA. Relationships between micronutrient intake and biochemical indicators of nutrient adequacy in a 'free-living' elderly UK population. Br J Nutr 1997; 77: 225-42.

33. Sharkey JR, Branch LG, Zohoori N, Giuliani C, BusbyWhitehead J, Haines PS. Inadequate nutrient intakes among homebound elderly and their correlation with individual characteristics and health-related factors. Am J Clin Nutr 2002; 76: 1435-45.

34. Ma J, Betts NM. Zinc and copper intakes and their major food sources for older adults in the 1994-96 continuing survey of food intakes by individuals (CSFII). J Nutr 2000; 130: 2838-43.

35. August D, Janghorbani M, Young VR. Determination of zinc and copper absorption at three dietary $\mathrm{Zn}-\mathrm{Cu}$ ratios by using stable isotope methods in young adult and elderly subjects. Am J Clin Nutr 1989; 50: 1457-63.

36. Turnlund JR, Durkin N, Costa F, Margen S. Stable isotope studies of zinc absorption and retention in young and elderly men. J Nutr 1986; 116: 1239-47.

37. Bales CW, Steinman LC, Freeland-Graves JH, Stone JM, Young RK. The effect of age on plasma zinc uptake and taste acuity. Am J Clin Nutr 1986; 44: 664-9.

38. Couzy F, Kastenmayer P, Mansourian R, Guinchard S, Munoz-Box R, Dirren H. Zinc absorption in healthy 
Prevalencia de deficiencia de Zn y Cu en adultos mayores - M. Olivares et al

elderly humans and the effect of diet. Am J Clin Nutr 1993; 58: 690-4.

39. Turnlund JR. Reager RD. Costa F. Iron and copper absorption in young and elderly men. Nutr Res 1988; 8: 333-43.

40. Haase H, Rink L. The immune system and the impact of zinc during aging. Immun Ageing 2009; 6: 9.

41. Percival SS. Copper and immunity. Am J Clin Nutr 1998; 67: 1064S-8S.

42. Caruso C, Buffa S, Candore G, Colonna-Romano G, Dunn-Walters D, Kipling D, et al. Mechanisms of immunosenescence. Immun Ageing 2009; 6: 10. 